\title{
Pulmonary Arteriovenous Malformation presenting as hemothorax in pregnancy: A case report.
}

\author{
Dinesh Chataut ${ }^{1}$, Sundar Suwal ${ }^{1}$, Kamal Subedi', Ajit Thapa ${ }^{1}$, Ram Kumar Ghimire ${ }^{2}$, Yadav Kumar \\ Deo Bhatta ${ }^{3}$. \\ ${ }^{1}$ Department of Radiology and Imaging, Tribhuvan University Teaching Hospital, Kathmandu, Nepal \\ ${ }^{2}$ Department of Radiology, Nepal Mediciti Hospital, Lalitpur, Nepal \\ ${ }^{3}$ Department of Cardiology, Shahid Gangalal National Heart Center, Kathmandu, Nepal
}

Correspondence: Dr. Sundar Suwal, Assistant Professor, Department of Radiology and Imaging, Tribhuvan University Teaching Hospital, Kathmandu, Nepal.

Email: sundarsuwal@iom.edu.np

\section{Abstract}

Introduction: Pulmonary arteriovenous malformation (PAVM) is a rare anomaly, mostly congenital, that tends to increase in size with the influence of various factors.

Case report: A primigravida at 37 weeks of gestation presented with acute onset of dyspnea due to right sided hemothorax. Computed tomography showed abnormal vessels with pseudoaneurysm in the right lower lobe and shunting between subsegmental branch of right descending pulmonary artery and right inferior pulmonary vein. Embolization of the lesion was planned after immediate cesarean section. Coil embolization with complete occlusion of supplying artery of the lesion was done.

Conclusion: PAVM, although rare can result in fatal complications if untreated. Less invasive image guided coil embolization is a promising treatment modality.

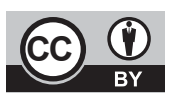

DOI: https://doi.org/10.3126/jssn.v23i1.33556

\section{Introduction}

Pulmonary arteriovenous malformation (PAVM) is a rare anomaly with low-resistance but high flow anomalous vessels due to abnormal communication between pulmonary arterial and venous systems with bypass of the capillary bed. Various other terminologies have been used for PAVM, like pulmonary arteriovenous fistula, pulmonary arteriovenous aneurysm and pulmonary angiomas. Most of these lesions are congenital malformations and acquired lesions are extremely uncommon. ${ }^{1}$ They tend to increase in size with influence of various factors like pulmonary arterial hypertension, puberty and pregnancy. ${ }^{2}$

\section{Case report}

A young 30 years old primigravida with 37 weeks gestation presented to Emergency Department with history of acute onset of shortness of breath for two to three hours. On examination, there was decreased breath sound on the right side of the chest. Immediate chest X-ray Posterior-Anterior view (PA view) was done which showed opaque right hemithorax suggesting right pleural effusion. Pleurocentesis was done in emergency department, which revealed hemorrhagic effusion. Contrast enhanced computed tomography (CT) with $\mathrm{CT}$ angiography was done which showed vascular malformation with pseudoaneurysm formation in the lower lobe of the right lung. Abnormal communication between the right descending pulmonary artery and right inferior pulmonary vein along the pseudoaneurysm was demonstrated. Associated mild to moderate right pleural fluid collection and adjacent basal atelectasis were seen (Figure 1). Patient was managed conservatively and planned for immediate cesarean section. As the patient was stable following the cesarean section, conventional pulmonary angiography with embolization was planned one-week post-partum.

JSSN 2020; 23 (1) 


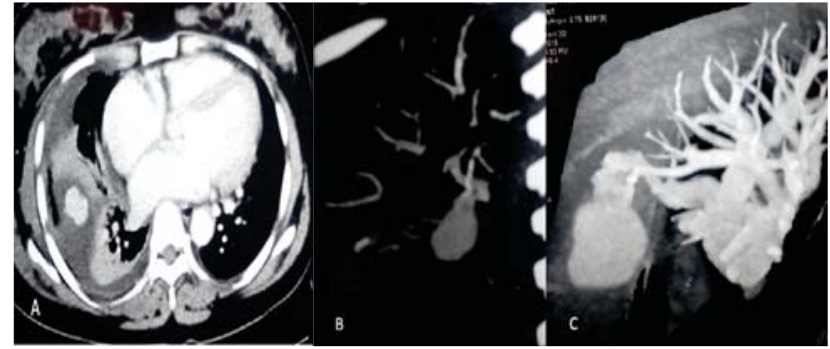

Figure 1. Contrast enhanced CT (A) showing contrast filled oval structure with adjacent atelectasis in right lower lobe and right pleural effusion. CT angiography (B and $\mathrm{C}$ ) showing shunting of branch of right descending pulmonary artery and right inferior pulmonary vein through the contrast filled structure.

Pre-anaesthetic check-up was done, and under local anesthesia, through right femoral vein approach, guide catheter was placed up to the distal end of Inferior Venacava (IVC). Swan Ganz catheter was introduced to pass into the pulmonary circulation. Guide wire was placed and then $5 \mathrm{~F}$ pigtail catheter was passed through it and selected angiography taken from the right descending pulmonary artery. Angiography showed pseudoaneurysm and abnormal tufts of vessels along subsegmental branch of right descending pulmonary artery, and high flow shunting with abnormal venous channels draining into right inferior pulmonary vein. Then $5 \mathrm{~F}$ multipurpose catheter was placed into the supplying pulmonary artery over the guidewire. Three coils of 0.35 inch, $5 \mathrm{mmx} 5 \mathrm{~cm}$ coil were placed which caused complete occlusion of the communicating channel. Angiography taken after the coil placement showed complete occlusion of the supplying artery (Figure 2). She was followed up after six months of embolization and the period was uneventful.

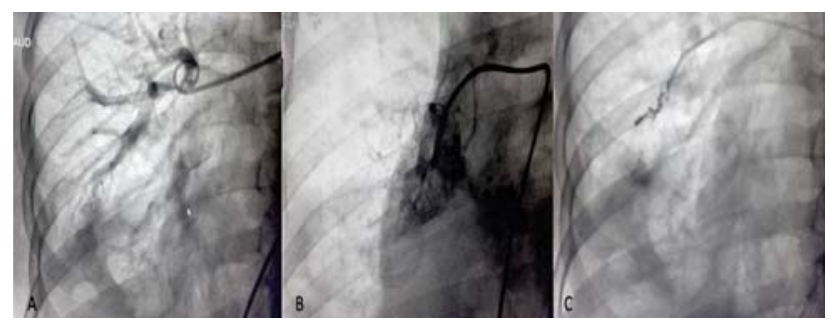

Figure 2. Right pulmonary artery angiogram (A) showing abnormal contrast filled outpouching in right lower lung supplied by branch of right descending pulmonary artery. Selective catheterization and angiogram (B) showing abnormal contrast blush and shunting of pulmonary artery and vein (pulmonary arteriovenous malformation). Selective angiogram of right descending pulmonary after coil embolization (C) showing complete occlusion of the artery feeding the malformation.

Journal of Society of Surgeons of Nepal (JSSN)

\section{Discussion}

Incidence of PAVM is around 2.5/100,000 with male to female ratio of $1.5-1.8: 1$. Although $80 \%$ of them are congenital, only about $10 \%$ are detected in childhood. ${ }^{2,3}$ Among the congenital cases, $70 \%$ of them are associated with hereditary hemorrhagic telangiectasia (HHT).,5 However, no obvious features of HHT were seen in our case.

PAVM can be asymptomatic with the incidental finding in chest $\mathrm{x}$-ray. Symptomatic cases usually present at fourth to sixth decades when the shunted blood volume is more than $25 \%$ of the total blood volume. They may present with cyanosis, dyspnea and hemoptysis as well. It may even present as cardiac or respiratory failure and its rupture resulting in hemothorax or rarely air embolism. PAVM rupture occurs irrespective of the lesion size and can occur at any age. ${ }^{4}$ Non-thoracic complications can be its presentation as well, such as stroke and brain abscess due to paradoxical embolism (right to left shunting). The presentation can be early in case of HHT or with influence of other factors likely pregnancy and pulmonary arterial hypertension. During pregnancy, complication rates increase with increased gestational age. ${ }^{2,4}$ Our case presented with hemothorax due to rupture of the vascular malformation during the third trimester of pregnancy.

Around $80 \%$ of PAVM are simple type with single feeding artery and draining vein. Complex cases have either two or more feeding arteries or two or more draining veins or both. ${ }^{4}$ Our case was simple PAVM. More than $50 \%$ of the lesions are seen in lower lobes as in our case. Lesions in upper lobes are least common. PAVM can be single or multiple (common with HHT); if multiple it can be unilateral or bilateral. ${ }^{1}$ Our case had a single simple PAVM in right lower lobe.

Usually, chest x-ray is the initial imaging modality for symptomatic cases. In asymptomatic cases also, it may be incidentally detected in chest $\mathrm{x}$-ray as round to oval opacity with branching afferent and efferent vessels. However, most of the times, chest $\mathrm{x}$-ray picture is non-specific with less obvious feeding and draining vessels. Common differential diagnoses based on chest x-ray findings of round to oval opacity can be calcified granuloma, bronchocele, neoplasm, pulmonary artery aneurysm and pulmonary vein varix. ${ }^{1}$ Chest $\mathrm{x}$-ray finding can be pleural fluid collection (hemothorax following its rupture) as well, like in our case. Due to sudden onset of dyspnea and hemothorax in our young lady without history of trauma, vascular malformation was suspected and contrast CT was advised to find the source of bleeding. Contrast enhanced computed

JSSN 2020; 23 (1) 
tomography (CT) is second line of investigation in these cases which can accurately show feeding arteries and draining veins. The feeding arteries most commonly arises from the pulmonary arteries, however, systemic arteries like bronchial artery, intercoastal artery, internal mammary artery or phrenic artery can also contribute to the feeders. Typically, drainage of the lesion is from pulmonary vein, but rarely it may drain into left atrium or inferior venacava. ${ }^{1}$ Transthoracic contrast echocardiography (TTCE) is gaining popularity for diagnosis of PAVM and it is comparable to CT even in calculating shunt size. ${ }^{3}$ However, TTCE is not widely and easily available as compared to CT, especially in underdeveloped countries.

If left untreated PAVM can be associated with various lifethreatening intrathoracic and extra-thoracic complications. Mortality can exceed $11 \%$. Thus even asymptomatic PAVM should be treated if the lesion size is more than two centimeters, or if the feeding artery is more than two millimeters in caliber. Symptomatic PAVM needs treatment irrespective of the lesion size and the size of feeding artery. ${ }^{4}$

Treatment modality in PAVM can be either surgery or embolotherapy or hormonal therapy. Image guided percutaneous embolization of the malformation is preferred as it is less invasive and there is no risk of anesthesia related complications. ${ }^{2}$ Also, it is successful in majority of patients. ${ }^{6,7}$ The aim of embolotherapy should be to occlude all malformations by selective catheterization of feeding pulmonary arteries. Embolic devices commonly used are coils, plugs and detachable balloons., ${ }^{2,7}$ In our case, we did coil embolization of the PAVM.

Most of the complications of embolotherapy are minor procedure-related complications like mild contrast allergy and local hematoma at puncture site. Pleurisy is also one of the common complications following embolotherapy, mainly with larger PAVM. Rarely, coil migration resulting in paradoxical coil embolism can occur. ${ }^{7}$ None of these complications were seen in our case.

\section{Conclusion}

Pulmonary arteriovenous malformation is a rare entity which can result in life-threatening complications if untreated. Chest x-ray is the initial imaging modality, however contrast enhanced $\mathrm{CT}$ or contrast echocardiography are preferred imaging modalities for planning its management. Percutaneous transcatheter embolization is a less invasive and very successful treatment modality.

\section{References}

1. Saboo SS, Chamarthy M, Bhalla S, Park H, Sutphin P, Kay F, et al. Pulmonary arteriovenous malformations: diagnosis. Cardiovasc Diagn Ther. 2018 Jun;8(3):325-37.

2. Sharma P, Kochar P, Sharma S, Gupta N, Li S, Hooda K, et al. A case of pulmonary arteriovenous malformation: role of interventional radiology in diagnosis and treatment. Ann Transl Med. 2017 Sep;5(17):345.

3. Sladden D, Casha A, Azzopardi C, Manche A. A large pulmonary arteriovenous malformation causing cerebrovascular accidents. BMJ Case Rep. 2015; 2015:bcr2014207786.

4. Navratil M, Vidjak V, Rubic F, Damir E, Turkalj M. Pulmonary arteriovenous malformations presenting as difficult-to-control asthma: a case report. J Med Case Rep. 2013;7:32.

5. Mangla A, Nevares A, Yamani N, Ansari N. Pulmonary arteriovenous malformation: a rare cause of dyspnoea on exertion. BMJ Case Rep. 2014 Apr 17;2014:bcr2014204087.

6. Shin JH, Park SJ, Ko G-Y, Yoon H-K, Gwon D-I, Kim J-H, et al. Embolotherapy for Pulmonary Arteriovenous Malformations in Patients without Hereditary Hemorrhagic Telangiectasia. Korean J Radiol. May-Jun 2010;11(3):312-9.

7. Iqbal M, Rossoff LJ, Steinberg HN, Marzouk KA, Siegel DN. Pulmonary arteriovenous malformations: a clinical review. Postgrad Med J. 2000 Jul;76(897):390-4. 